malignancies, usually seen in peri- or post-menopausal women. Criteria for diagnosis of fibrosarcoma include mitotic count, the most important feature for distinguishing between benign and malignant lesions. Only few cases have been reported due to its low incidence and poor prognosis. These tumors are easily misdiagnosed so sometimes diagnosis is primarily based on histopathological and immunohistochemistry (IHC) report as in our case report. We report a rare case of ovarian fibrosarcoma. Methods: A 40 year, P5L5, woman visited to gynecological oncology department of IGIMS, Patna, with complain of abdominal distention for 6 months. Her menstrual history was regular with average flow. On abdominal examination-a lump of 32 week size with mobility found. On vaginal examinationcervix was healthy, uterine size could not be assessed due to such huge mass. Her tumor markers showed raised cancer antigen 125 level with value of 152.7. Other tumor markers were within normal limit. Computed tomography abdomen showed $22 \times 19 \times 16 \mathrm{~cm}$ mass in left adenexa with multiple septae and few cystic areas.

Results: Primary debulking surgery done. Final pathological diagnosis was fibrosarcoma of left ovary. IHC showed ki-67-35\%. Conclusion: Primary ovarian fibrosarcomas is a rare neoplasm of ovary but it must be considered as differential diagnosis of unilateral huge solid ovarian mass in all age group. ki-67 along with mitosis, greater than 4 mitotic areas/HPF, has recently become an important indicator for diagnosis of ovarian fibrosarcoma. Preoperative diagnosis is often difficult. Gynaeoncologist has to wait for histopathological and IHC report for final diagnosis.

Poster (025)

Rare Tumors \& Metastatic Tumors

https://doi.org/10.3802/jgo.2021.32.S1.025

\section{Mature cystic teratoma of the ovary with keratinizing squamous cell carcinoma}

\section{Renardy Reza Razali, ' Kartiwa Hadi Nurianto,} Primariadewi Rustamadji

Faculty of Medicine, Indonesia University, Jakarta, Indonesia (renardireza@gmail.com)

Objective: Learning about clinical manifestation and diagnosis of mature teratoma cases with malignant transformation. Methods: A 41-year-old woman, P3A0, was referred from primary hospital because of post-operative pathological examination revealed a malignancy. Earlier, she underwent subtotal abdominal hysterectomy and bilateral salpingooophorectomy at primary hospital for indication abdominal enlargement and dysmenorrhea. After procedure, post- operative pathological result revealed; keratinized squamous cell carcinoma, poorly differentiated originating from mature teratoma of the right ovary. Tumor cell infiltration was seen in the tissue around the right tube.

Results: Histological according to keratinized squamous cell carcinoma, poorly differentiated originating from mature teratoma of the right ovary.

Conclusion: Malignant transformation of benign ovarian tumors, including mature teratoma, is very rare. In addition, the clinical findings and pre-operative examinations did not help a lot to establish the diagnosis. Often the diagnosis was discovered unexpectedly in post-operative specimens such as happened in this case.

Poster (026)

Epithelial Ovarian Cancer including Borderline Tumor https://doi.org/10.3802/jgo.2021.32.S1.026

\section{Preoperative evaluation for prediction of suboptimal primary cytoreductive surgery in advanced-stage ovarian cancer}

Ruai Kittikhun, "Witsarut Apikijmeta, Suttida Intharaburan

Phramongkutklao Hospital, Bangkok, Thailand (ruai.kittikhun@gmail.com)

Objective: To identify the preoperative predicting factors of suboptimal primary cytoreductive surgery (PCS) in advanced stage ovarian cancer patients and to determine the suboptimal cytoreduction rate.

Methods: Retrospective cross-sectional study was conducted between 1 January 2014 to 31 December 2019. Ovarian cancer International Federation of Gynecology and Obstetrics (FIGO) stage III-IV patients who underwent PCS in Phramongkutklao Hospital were reviewed. Data collection included age, performance status, serum cancer-antigen 125 (CA125) and radiological criteria from computed tomographic (CT) scan of whole abdomen with intravenous and oral contrast provided within 30 days to PCS. Univariate and multivariate logistic regression analysis were employed to access the predicting factors of suboptimal PCS. The suboptimal cytoreduction rate was presented in percentage.

Results: Eighty patients with advanced ovarian cancer who had PCS by gynecologic oncologist in Phramongkutklao Hospital were enrolled. The majority of ovarian cancer FIGO stage in this population was IIIC (60\%). The suboptimal surgery rate in the study was $62.5 \%$. According to univariate analysis, Eastern Cooperative Oncology Group performance score $=1-3$ $(p=0.017)$, serum CA125 level of 500 units $/ \mathrm{mL}$ or more $(p=0.02)$, CT features of retroperitoneal lymph nodes above renal hilum 
$>1 \mathrm{~cm}(\mathrm{p}=0.008)$, diffuse small bowel adhesion/thickening $(\mathrm{p}=0.018)$ and lesser sac lesion $>1 \mathrm{~cm}(\mathrm{p}<0.001)$ were classified as predicting factors for suboptimal surgery. However, only CT features of retroperitoneal lymph nodes above renal hilum $>1 \mathrm{~cm}(\mathrm{p}=0.046)$ and lesser sac lesion $>1 \mathrm{~cm}(\mathrm{p}=0.004)$ were significant predicting factors for suboptimal surgery in multivariate analysis.

Conclusion: The preoperative predicting factors of suboptimal primary cytoreductive surgery in advanced stage ovarian cancer were retroperitoneal lymph nodes above renal hilum $>1 \mathrm{~cm}$ and lesser sac lesion $>1 \mathrm{~cm}$ on CT scan. The suboptimal cytoreduction rate was $62.5 \%$.

Poster (027)

Epithelial Ovarian Cancer including Borderline Tumor https://doi.org/10.3802/jgo.2021.32.S1.027

\section{Wip1 suggested as an independent predictor for poor overall survival through chemoresistance in specifically advanced- stage ovarian clear cell carcinomas}

\section{Chenyang Xu, Takeo Minaguchi, ' Nan Qi, Hiroya Itagaki, Ayumi Shikama, Nobutaka Tasaka, Azusa Akiyama, Sari Nakao, Hiroyuki Ochi, Toyomi Satoh \\ University of Tsukuba, Tsukuba, Japan (minaguchit@md.tsukuba.ac.jp)}

Objective: Although ovarian clear cell carcinoma (OCCC) tends to be diagnosed at an early stage, advanced-stage disease shows poor prognosis due to chemoresistance unlike the more common high-grade serous carcinoma.

Methods: We explored the differential roles of the Wip1-p38p53 DNA damage response pathway in early or advanced-stage OCCCs, respectively. We performed immunohistochemistry of Wip1, nuclear/cytoplasmic phospho-p38, p53 and phospho-p53 in OCCCs from consecutive 143 patients. Clinicopathological and prognostic data were retrospectively reviewed and correlated with the protein expressions.

Results: High Wip1 expression was significantly associated with positive $\mathrm{p} 53$, which was significantly associated with low nuclear phospho-p38 expression ( $\mathrm{p}=0.011$ and 0.0094 , respectively). In the early-stage diseases $(n=102)$, patients with positive $\mathrm{p} 53$ showed trends toward worse overall survival (OS) ( $p=0.062)$. Whereas in the advanced-stage diseases $(n=41)$, patients with highWip1 expression showed significantly worse OS ( $\mathrm{p}=0.0012$ ). The univariate and multivariate analyses for prognostic factors in the advanced-stage diseases indicated that high Wip1 expression was significant and independent for worse OS $(\mathrm{p}=0.011)$, but not in the early-stage diseases. Furthermore, high Wip1 showed a trend toward shorter treatment-free interval in advanced stages, but not in early stages ( $\mathrm{p}=0.083$ vs. $\mathrm{p}=0.93$ ). Conclusion: Wip1 appears to play a significant role for the prognosis of OCCCs through chemoresistance specifically in advanced stages, suggesting that Wip1 may serve as a reasonable therapeutic target for improving the poor prognosis of advanced-stage OCCC.

Poster (028)

Epithelial Ovarian Cancer including Borderline Tumor https://doi.org/10.3802/jgo.2021.32.S1.028

\section{Ambulatory chemotherapy in epithelial ovarian cancer patients}

\section{Suwanit Therasakvichya, ${ }^{*}$ Siree Limtiamchareon}

Faculty of Medicine, Siriraj Hospital, Mahidol University, Salaya, Thailand (suwanit.the@mahidol.ac.th)

Objective: Chemotherapy is a very effective for treatment of epithelial ovarian cancer (EOC) in not only primary but also in recurrence disease. According to health care policy to date, standard chemotherapy (paclitaxel plus carboplatin) should be managed as outpatient care. To determine the response rate and the toxicity of a 3-hour paclitaxel plus carboplatin administered as ambulatory chemotherapy for primary or recurrence EOC.

Methods: Retrospective review of medical records between April 2010 and July 2012. Seventy-nine EOC patients with mean age of 55 years old who were treated with adjuvant or salvage chemotherapy (intravenous paclitaxel $175 \mathrm{mg} / \mathrm{m}^{2}$ drip in 3 hours plus carboplatin area under the curve [AUC] 5 drip in 1 hour) every 21 days for 6 cycles.

Results: Twenty-four (36.4\%) patients were in early stage (I-II) and $42(63.6 \%)$ patients were in advanced stage (III-IV). Of $83.5 \%$ of the patients were in primary treatment and $16.5 \%$ were in recurrence. The complete response rates were $79.2 \%$, $64.3 \%$, and $46.2 \%$ in early, advanced, and recurrence cases, respectively. Four patients had hypersensitivity reaction during the first administration of paclitaxel, another patient had hypersensitivity reaction after carboplatin infusion. Only 3.8\% (3/79) developed febrile neutropenia which were manageable. Fourteen patients $(17.7 \%)$ had grade III-IV neutropenia without any symptoms. Most of the patients complained of grade I neuropathy.

Conclusion: Three-hour $175 \mathrm{mg} / \mathrm{m}^{2}$ paclitaxel plus 1 hour carboplatin AUC 5 is effective and quite safe for ambulatory care. However, hypersensitivity reaction should be accounted for especially during the first course of administration. 